\title{
Diferença na degeneração articular de acordo com o tipo de esporte*
}

\section{Difference in Articular Degeneration Depending on the Type of Sport}

\author{
Pedro Baches Jorge ${ }^{1}$ Jan Willem Cerf Sprey ${ }^{1}$ Guilherme Morgado Runco ${ }^{1}$ Marcos Vaz de Lima ${ }^{1}$ \\ Nilson Roberto Severino ${ }^{1}$ Claudio Santili ${ }^{1}$ \\ 1 Grupo de Trauma do Esporte, Departamento de Ortopedia e \\ Traumatologia, Faculdade de Ciências Médicas, Irmandade de \\ Misericórdia da Santa Casa de São Paulo, São Paulo, SP, Brasil \\ Endereço para correspondência Pedro Baches Jorge, MD, \\ Clínica SO.U - Matriz, Rua Barata Ribeiro, 398, $3^{\circ}$ andar, Bela Vista, \\ São Paulo, 01308-000, SP, Brasil (e-mail: pbj1980@hotmail.com).
}

Rev Bras Ortop 2019;54:509-515.

\section{Resumo \\ Palavras-chave \\ - cartilagem \\ - biomarcadores \\ - artrose \\ - atletas \\ Objetivo Determinar se a degradação de colágeno tipo II é determinada pelo tipo de esporte. O telopeptídeo carboxiterminal do colágeno tipo II (CTX-II), biomarcador de soro de degradação de colágeno, foi medido em atletas de esportes diferentes e comparado aos controles correspondentes. \\ Métodos A amostra consistiu em 70 participantes do sexo feminino com idade entre 18 a 25 anos, das quais 15 eram membros de uma equipe de futebol, 10 de uma equipe de futebol de salão, 10 de uma equipe de handebol, 18 de uma equipe de voleibol, e 7 de uma equipe de natação. Foram incluídos no grupo de controle 9 indivíduos sedentários, pareados por idade e sexo. Uma amostra de sangue de $3 \mathrm{ml}$ foi coletada de cada participante e analisada usando um ensaio imunossorvente ligado a enzima (ELISA, do inglês enzyme-linked immunosorbent assay). \\ Resultados Uma comparação das concentrações de CTX-II das praticantes de dife- rentes esportes em comparação com o grupo de controle apresentou os seguintes valores de $p$ : voleibol $(p=0,21)$; futebol $(p=0,91)$; handebol $(p=0,13)$; futebol de salão $(p=0,02)$ e natação $(p=0,0015)$. Portanto, na população investigada, o futebol de salão apresentou o maior risco de degradação do colágeno tipo II, e, consequente- mente, de degradação da cartilagem articular, enquanto a prática de natação foi um fator protetor para a cartilagem articular. Não foi observada diferença estatisticamente significativa no índice de massa corporal entre os grupos. \\ Conclusão As jogadoras de futebol de salão estão expostas a uma maior degradação articular, enquanto as nadadoras apresentam menor degradação da cartilagem em comparação com o grupo de controle na população estudada, o que sugere que o fortalecimento dos músculos periarticulares e o exercício aeróbico em ambientes de baixa carga têm um efeito positivo na cartilagem articular.}

Published Originally by Elsevier Editora Ltda.

recebido

31 de Outubro de 2017

aceito

21 de Fevereiro de 2018
DOI https://doi.org/

10.1016/j.rboe.2018.02.012. ISSN 0102-3616.
Copyright $\odot 2019$ by Sociedade Brasileira License terms de Ortopedia e Traumatologia. Published by Thieme Revnter Publicações Ltda, Rio de Janeiro, Brazil 


\begin{abstract}
Objective To determine whether type-II collagen degradation is determined by the type of sport. Carboxy-terminal telopeptide of type-II collagen (CTX-II), a serum biomarker of collagen degradation, was measured in athletes who play different sports, and was compared with matched controls.

Methods The sample size consisted of 70 female participants aged between 18 and 25 years, 15 of whom were members of a soccer team, 10 of a futsal (a variant of association football played on a hard court) team, 10 of a handball team, 18 of a volleyball team, and 7 of a swimming team. A total of 9 age- and sex-matched individuals with sedentary lifestyles were included in the control group. 3-mL blood samples were collected from each participant, and they were analyzed using an enzyme-linked immunosorbent assay (ELISA).

Results A comparison of the CTX-II concentrations of the players of different sports with those of the control group resulted in the following $p$-values: volleyball $(p=0.21)$; soccer $(p=0.91)$; handball $(p=0.13)$; futsal $(p=0.02)$; and swimming $(p=0.0015)$. Therefore, in the investigated population, futsal represented the highest risk for type-II collagen degradation and, consequently, for articular cartilage degradation, whereas swimming was a protective factor for the articular cartilage. No statistically significant

Keywords

- cartilage

- biomarkers

- arthrosis

- athletes difference was found in the body mass index among the groups.

Conclusion Futsal players are exposed to greater articular degradation, while swimmers exhibited less cartilage degradation compared with the control group in the study population, suggesting that strengthening the periarticular muscles and aerobic exercise in low-load environments has a positive effect on the articular cartilage.
\end{abstract}

\section{Introdução}

A cartilagem articular é um tecido aneural e avascular especializado, que cobre as partes ósseas das articulações diartrodiais. Sua função é facilitar o movimento suave por meio de seu coeficiente de baixa fricção, além de absorver choques e aguentar cargas de apoio em vários planos. ${ }^{1,2}$

A preservação da cartilagem articular depende da manutenção da integridade de sua estrutura molecular. ${ }^{1}$ As principais macromoléculas que compõem a cartilagem são colágeno e proteoglicanos; durante o curso da vida, a atividade das células condrais é determinada por vários fatores autócrinos e não autócrinos, que resultam na manutenção ou destruição da homeostase articular. A osteoartrose é uma condição comum em idosos, mas também pode afetar pessoas jovens que estão sujeitas a cargas articulares excessivas. ${ }^{1-17}$

Em adultos, a ativação da função articular dentro dos limites fisiológicos de carga e frequência de trabalho é crucial para manter a saúde das articulações: ${ }^{3-6,9,13}$ portanto, articulações trabalhando abaixo dos níveis exigidos também correm o risco de degenerar. ${ }^{10}$

Frequência de trabalho e sobrecarga articular são fatores importantes na destruição articular, que é caracterizada por danos ao tecido cartilaginoso. ${ }^{2}$ De fato, a exposição excessiva à sobrecarga leva ao desgaste articular precoce, ${ }^{2-6,9,10,13}$ que é então perpetuado por uma cascata inflamatória que afeta todo o tecido articular. ${ }^{16}$ Portanto, atletas de alto desempenho submetidos a cargas de treinamento excessivas durante um curto período de tempo correm maior risco de desenvolver dano articular; os joelhos, em particular, tendem a ser supe- rexpostos na maioria dos esportes. Em função da demanda constante por desempenho máximo, o desgaste articular precoce e as deficiências funcionais podem resultar no fim de uma carreira atlética.

A maioria dos pacientes é diagnosticada nos estágios avançados da artrose, quando uma série de eventos metabólicos já ocorreu, e o processo provavelmente já passou do ponto em que as intervenções farmacológicas e cirúrgicas são realmente eficazes. ${ }^{18}$

Os produtos da degradação da matriz de colágeno podem servir como marcadores úteis da gravidade e progressão da artrose. Tais produtos podem ser medidos no fluido sinovial, no sangue ou na urina, e, assim, fornecer informações importantes para o diagnóstico precoce da artrose.

\section{Quanto ao Colágeno Articular}

O colágeno representa 50 a $60 \%$ do peso seco da cartilagem. Suas fibras formam uma rede densa que dá forma a esse tecido. As propriedades mecânicas mais importantes do colágeno são a resistência e a resiliência, que são transmitidas para a cartilagem. ${ }^{1}$

O colágeno tipo II é específico da cartilagem, e representa quase $98 \%$ do conteúdo total de colágeno nesse tecido. ${ }^{1}$

O colágeno tipo II é a maior macromolécula da qual a cartilagem é composta, e consiste em três cadeias polipeptídicas idênticas dispostas em uma hélice tripla. Cada uma dessas cadeias é sintetizada como uma pró-cadeia que contém grandes pró-peptídeos nas suas extremidades, que são separados da parte central por telopeptídeos. Durante a maturação das moléculas de colágeno tipo II, as proteases clivam os pró- 
peptídeos; assim, a estrutura madura consiste na parte central da tripla hélice e telopeptídeos.

Quando os componentes articulares se degradam, são expelidos do tecido de origem e medidos com maior precisão no fluido articular. No entanto, ao estudar a osteoartrite, a medição de biomarcadores no sangue e na urina é feita por métodos menos agressivos e que ainda são eficazes e precisos. ${ }^{1}$

O telopeptídeo carboxiterminal de colágeno do tipo II (CTX-II) é um biomarcador de degradação articular. O uso de CTX-II como biomarcador, além ser feito por conta de sua relação direta com o grau radiológico da doença, escores clínicos e gravidade das lesões cartilaginosas, também está bem estabelecido na literatura. ${ }^{2,18-28}$ Assim, a medida do CTX-II parece ser um método eficaz para investigar a renovação do colágeno tipo II.

Este estudo teve como objetivo detectar a degradação precoce do colágeno tipo II articular, medindo CTX-II no sangue de atletas do sexo feminino que praticam esportes diferentes, e que foram comparadas com um grupo de controle. Procuramos estabelecer se a participação esportiva é um fator de risco para a degradação articular precoce em nosso país, e qual esporte foi o mais prejudicial ao colágeno tipo II articular na população investigada.

\section{Materiais e Métodos}

Este estudo foi realizado no Grupo de Trauma Esportivo do departamento de ortopedia da nossa instituição. 0 estudo foi aprovado pelo comitê de ética institucional (número 244/11), sendo que todos os participantes assinaram termos de consentimento livre e esclarecido.

A população do estudo consistia apenas de mulheres entre 18 e 25 anos, integrantes de equipes esportivas competitivas, incluindo futebol, futsal, handebol, voleibol e natação, no Clube Atlético São José, parceiro do Grupo de Trauma Esportivo da nossa instituição. Todqs as participantes tinham que ter de 5 a 8 anos de treinamento no esporte, e nenhuma das atletas praticava outros esportes.

Pacientes com dor articular em tratamento, histórico de cirurgia ortopédica articular ou de dor articular não tratada devido a qualquer causa foram excluídas do estudo.

Um total de indivíduos sedentários, que preencheram os critérios de inclusão, formaram o grupo de controle. Nenhum deles havia praticado de esportes de maneira competitiva.

Um total de 70 mulheres com idade entre 18 e 25 anos foram incluídas, e separadas pelo tipo de esporte que praticavam: futebol $(n=15)$, futsal $(n=10)$, handebol $(n=10)$, voleibol $(n=18)$ e natação $(n=7)$; 9 indivíduos sedentários foram incluídos no grupo de controle.

Um total de $3 \mathrm{ml}$ de sangue foi coletado de cada participante por punção venosa simples do membro superior não dominante, utilizando um kit de coleta a vácuo, no qual há contato da amostra com ácido etilenodiamino tetra-acético (AEDT), o que não afeta o resultado, e o índice de massa corporal (IMC) foi calculado pelo peso dividido pelo quadrado da altura de cada pessoa.

O sangue foi centrifugado, e o plasma foi armazenado a - 80॰C até que todas as amostras de sangue estivessem prontas para análise, por um total de até 23 dias, porque o teste foi realizado em um único dia. As amostras foram analisadas para detectar CTX-II humano utilizando ensaio imunossorvente ligado a enzima (teste do tipo ELISA, do inglês enzyme-linked immunosorbent assay; kit Hu CTX-II, Cusabio Biotech, Houston, TX, EUA). Este teste tem $100 \%$ de especificidade para o CTX-II humano, sem qualquer reação cruzada, com um nível mínimo de detecção de $0,2 \mathrm{ng} / \mathrm{ml}$, o que é informado pelo fabricante do kit.

Os resultados foram comparados pelo teste $t$ de Student com intervalo de confiança de 95\% (IC95\%), no qual valores de $p<0,05$ foram considerados estatisticamente significativos.

\section{Resultados}

Um total de 69 mulheres com idade entre 18 e 25 anos foram incluídas e divididas conforme o esporte que praticavam: futebol $(n=15)$, futsal $(n=10)$, handebol $(n=10)$, voleibol $(\mathrm{n}=18)$ e natação $(\mathrm{n}=7)$; 9 indivíduos sedentários foram incluídos no grupo de controle.

A idade média para o grupo de controle foi de 22,6 anos, a média do IMC foi de 22,3, e a média da concentração do CTXII foi de $0,453 \mathrm{ng} / \mathrm{mL}$ (- Tabela $\mathbf{1}$ ).

Entre as 18 jogadoras de voleibol, a idade média foi de 18,3 anos, a média do IMC foi de 22,33, e a média do CTX-II foi de $0,429 \mathrm{ng} / \mathrm{ml}$; essa equipe treinava 36 horas por semana (-Tabela 2).

Entre as 15 jogadoras de futebol, a idade média foi de 22,36 anos, a média do IMC foi de 22,06, e a média do CTX-II foi de $0,456 \mathrm{ng} / \mathrm{ml}$; essa equipe treinava 15 horas por semana (-Tabela 3).

Entre as jogadoras de futsal, a idade média foi de 18,5 anos, a média do IMC foi de 22,21, e a média do CTX-II foi de $0,542 \mathrm{ng} / \mathrm{ml}$; essa equipe treinava 15 horas por semana

\section{(-Tabela 4).}

Entre as jogadoras de handebol, a idade média foi de 18,9 anos, a média do IMC foi de 22,88 , e a média do CTX-II foi de $0,416 \mathrm{ng} / \mathrm{ml}$; essa equipe treinava 25 horas por semana (-Tabela 5).

Entre as nadadoras, a média de idade foi de 18,9 anos, a média do IMC foi de 20,71, e a média do CTX-II foi de 0,373 ng/ $\mathrm{ml}$; essa equipe treinava 12 horas por semana (-Tabela 6 ).

Tabela 1 Grupo de controle

\begin{tabular}{|l|l|l|l|l|l|}
\hline Participante & $\begin{array}{l}\text { Idade } \\
\text { (anos) }\end{array}$ & $\begin{array}{l}\text { Altura } \\
\text { (metros) }\end{array}$ & $\begin{array}{l}\text { Peso } \\
\text { (quilos) }\end{array}$ & IMC & $\begin{array}{l}\text { CTX-II } \\
\text { (ng/ml) }\end{array}$ \\
\hline 1 & 22 & 1,7 & 48 & 16,6 & 0,486 \\
\hline 2 & 21 & 1,7 & 65 & 22,49 & 0,427 \\
\hline 3 & 22 & 1,5 & 63 & 28 & 0,443 \\
\hline 4 & 24 & 1,64 & 50 & 18,59 & 0,461 \\
\hline 5 & 22 & 1,71 & 64 & 21,88 & 0,474 \\
\hline 6 & 23 & 1,57 & 53 & 21,5 & 0,45 \\
\hline 7 & 25 & 1,7 & 72 & 24,91 & 0,442 \\
\hline 8 & 22 & 1,68 & 53 & 18,77 & 0,432 \\
\hline 9 & 22 & 1,59 & 67 & 26,5 & 0,459 \\
\hline
\end{tabular}

Abreviaturas: CTX-II, telopeptídeo carboxiterminal do colágeno tipo II; IMC, índice de massa corporal. 
512 Diferença na degeneração articular de acordo com o tipo de esporte Jorge et al.

Tabela 2 Voleibol

\begin{tabular}{|l|l|l|l|l|l|}
\hline Atleta & $\begin{array}{l}\text { Idade } \\
\text { (anos) }\end{array}$ & $\begin{array}{l}\text { Altura } \\
\text { (metros) }\end{array}$ & $\begin{array}{l}\text { Peso } \\
\text { (quilos) }\end{array}$ & IMC & $\begin{array}{l}\text { CTX-II } \\
\text { (ng/ml) }\end{array}$ \\
\hline 10 & 18 & 1,85 & 80 & 23,37 & 0,421 \\
\hline 11 & 18 & 1,71 & 74 & 25,3 & 0,418 \\
\hline 12 & 18 & 1,79 & 66,6 & 20,78 & 0,426 \\
\hline 13 & 19 & 1,8 & 70 & 21,6 & 0,424 \\
\hline 14 & 19 & 1,83 & 75 & 22,39 & 0,637 \\
\hline 15 & 20 & 1,86 & 66 & 19,07 & 0,449 \\
\hline 16 & 20 & 1,82 & 67,5 & 20,37 & 0,443 \\
\hline 17 & 18 & 1,66 & 54 & 19,59 & 0,407 \\
\hline 18 & 18 & 1,77 & 66 & 21,06 & 0,615 \\
\hline 19 & 19 & 1,86 & 75 & 21,67 & 0,451 \\
\hline 20 & 23 & 1,73 & 83 & 27,73 & 0,509 \\
\hline 21 & 18 & 1,79 & 86 & 26,84 & 0,506 \\
\hline 22 & 18 & 1,62 & 52 & 19,81 & 0,577 \\
\hline 23 & 18 & 1,79 & 70 & 21,84 & 0,591 \\
\hline 24 & 18 & 1,82 & 71 & 21,43 & 0,434 \\
\hline 25 & 18 & 1,86 & 80 & 23,12 & 0,418 \\
\hline 26 & 18 & 1,75 & 68 & 22,2 & 0,429 \\
\hline 27 & 18 & 1,62 & 61 & 23,24 & 0,429 \\
\hline
\end{tabular}

Abreviaturas: CTX-II, telopeptídeo carboxiterminal do colágeno tipo II; IMC, índice de massa corporal.

Tabela 3 Futebol

\begin{tabular}{|l|l|l|l|l|l|}
\hline Atleta & $\begin{array}{l}\text { Idade } \\
\text { (anos) }\end{array}$ & $\begin{array}{l}\text { Altura } \\
\text { (metros) }\end{array}$ & $\begin{array}{l}\text { Peso } \\
\text { (quilos) }\end{array}$ & IMC & $\begin{array}{l}\text { CTX-II } \\
\text { (ng/ml) }\end{array}$ \\
\hline 28 & 25 & 1,61 & 52 & 20,06 & 0,526 \\
\hline 29 & 24 & 1,67 & 74 & 26,53 & 0,498 \\
\hline 30 & 18 & 1,75 & 60 & 19,59 & 0,466 \\
\hline 31 & 21 & 1,62 & 59 & 22,48 & 0,499 \\
\hline 32 & 19 & 1,68 & 56 & 17,84 & 0,527 \\
\hline 33 & 20 & 1,56 & 52 & 21,37 & 0,495 \\
\hline 34 & 25 & 1,68 & 70 & 24,8 & 0,476 \\
\hline 35 & 24 & 1,57 & 57 & 23,12 & 0,475 \\
\hline 36 & 22 & 1,63 & 62 & 23,33 & 0,496 \\
\hline 37 & 22 & 1,6 & 57 & 22,26 & 0,46 \\
\hline 38 & 24 & 1,54 & 50 & 21,08 & 0,441 \\
\hline 39 & 25 & 1,7 & 67 & 23,18 & 0,431 \\
\hline 40 & 19 & 1,65 & 65 & 23,87 & 0,136 \\
\hline 41 & 19 & 1,76 & 60 & 19,36 & 0,136 \\
\hline 42 & 21 & 1,62 & 62 & 23,33 & 0,507 \\
\hline
\end{tabular}

Abreviaturas: CTX-II, telopeptídeo carboxiterminal do colágeno tipo II; IMC, índice de massa corporal.

Os seguintes valores de $p$ foram obtidos comparando as atletas dos diferentes esportes ao grupo de controle usando o teste $t$ de Student: voleibol, $p=0,21$ ( - Fig. 1); futebol, $p=0,91$ (-Fig. 2); handebol, $p=0,13$ (-Fig. 3); futsal, $p=0,02$ (-Fig. 4); e natação, $p=0,0015$ (-Fig. 5).

Assim, o futsal representou o maior risco para a degradação do colágeno tipo II, e, consequentemente, para a degradação da
Tabela 4 Futsal

\begin{tabular}{|l|l|l|l|l|l|}
\hline Atleta & $\begin{array}{l}\text { Idade } \\
\text { (anos) }\end{array}$ & $\begin{array}{l}\text { Altura } \\
\text { (metros) }\end{array}$ & $\begin{array}{l}\text { Peso } \\
\text { (quilos) }\end{array}$ & IMC & $\begin{array}{l}\text { CTX-II } \\
\text { (ng/ml) }\end{array}$ \\
\hline 43 & 18 & 1,65 & 59 & 21,67 & 0,512 \\
\hline 44 & 18 & 1,73 & 59 & 19,71 & 0,475 \\
\hline 45 & 18 & 1,55 & 45 & 18,73 & 0,552 \\
\hline 46 & 18 & 1,6 & 60 & 23,44 & 0,643 \\
\hline 47 & 19 & 1,7 & 68 & 23,53 & 0,473 \\
\hline 48 & 18 & 1,59 & 60 & 23,73 & 0,591 \\
\hline 49 & 20 & 1,67 & 65 & 23,31 & 0,771 \\
\hline 50 & 19 & 1,58 & 52 & 20,83 & 0,522 \\
\hline 51 & 19 & 1,62 & 64 & 24,39 & 0,445 \\
\hline 52 & 18 & 1,49 & 50 & 22,71 & 0,431 \\
\hline
\end{tabular}

Abreviaturas: CTX-II, telopeptídeo carboxiterminal do colágeno tipo II; IMC, índice de massa corporal.

Tabela 5 Handebol

\begin{tabular}{|l|l|l|l|l|l|}
\hline Atleta & $\begin{array}{l}\text { Idade } \\
\text { (anos) }\end{array}$ & $\begin{array}{l}\text { Altura } \\
\text { (metros) }\end{array}$ & $\begin{array}{l}\text { Peso } \\
\text { (quilos) }\end{array}$ & IMC & $\begin{array}{l}\text { CTX-II } \\
\text { (ng/ml) }\end{array}$ \\
\hline 53 & 19 & 1,61 & 56 & 21,6 & 0,419 \\
\hline 54 & 18 & 1,71 & 71 & 24,28 & 0,439 \\
\hline 55 & 18 & 1,75 & 69 & 22,53 & 0,451 \\
\hline 56 & 18 & 1,67 & 48 & 17,21 & 0,41 \\
\hline 57 & 21 & 1,69 & 83 & 29,06 & 0,522 \\
\hline 58 & 19 & 1,68 & 69 & 24,44 & 0,297 \\
\hline 59 & 18 & 1,6 & 55 & 21,48 & 0,471 \\
\hline 60 & 19 & 1,82 & 72 & 21,73 & 0,332 \\
\hline 61 & 21 & 1,7 & 65 & 22,49 & 0,358 \\
\hline 62 & 18 & 1,71 & 70 & 23,93 & 0,458 \\
\hline
\end{tabular}

Abreviaturas: CTX-II, telopeptídeo carboxiterminal do colágeno tipo II; IMC, índice de massa corporal.

Tabela 6 Natação

\begin{tabular}{|l|l|l|l|l|l|}
\hline Atleta & $\begin{array}{l}\text { Idade } \\
\text { (anos) }\end{array}$ & $\begin{array}{l}\text { Altura } \\
\text { (metros) }\end{array}$ & $\begin{array}{l}\text { Peso } \\
\text { (quilos) }\end{array}$ & IMC & $\begin{array}{l}\text { CTX-II } \\
\text { (ng/ml) }\end{array}$ \\
\hline 63 & 18 & 1,73 & 63 & 21,04 & 0,345 \\
\hline 64 & 18 & 1,64 & 54 & 20,07 & 0,365 \\
\hline 65 & 19 & 1,63 & 55 & 20,70 & 0,45 \\
\hline 66 & 19 & 1,68 & 57 & 20,19 & 0,323 \\
\hline 67 & 18 & 1,71 & 61 & 20,86 & 0,356 \\
\hline 68 & 19 & 1,69 & 60 & 21 & 0,371 \\
\hline 69 & 21 & 1,74 & 64 & 21,13 & 0,402 \\
\hline
\end{tabular}

Abreviaturas: CTX-II, telopeptídeo carboxiterminal do colágeno tipo II; IMC, índice de massa corporal.

cartilagem articular, enquanto a natação foi um fator de proteção para a cartilagem articular na população investigada.

\section{Discussão}

Atualmente, a prevenção é enfatizada ao longo do tratamento; assim, o diagnóstico precoce da osteoartrose é de 
Diferença na degeneração articular de acordo com o tipo de esporte Jorge et al. 513

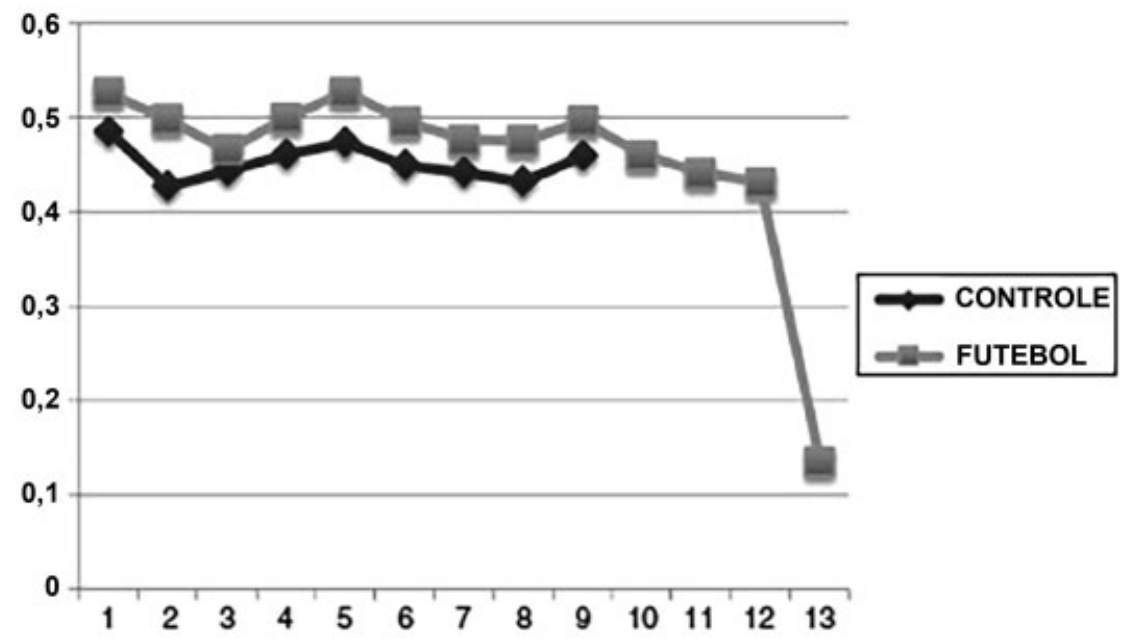

Fig. 1 Comparação dos resultados dos níveis de telopeptídeo carboxiterminal do colágeno tipo II (CTX-II) entre o grupo de controle e as jogadoras de futebol.

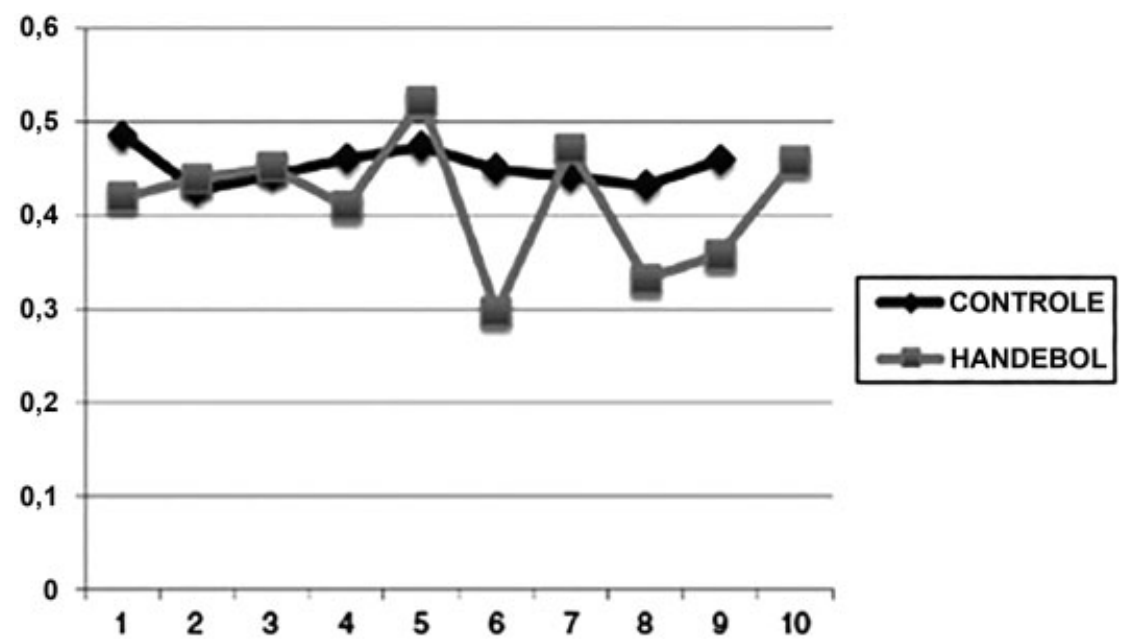

Fig. 2 Comparação dos resultados dos níveis de CTX-II entre o grupo de controle e as jogadoras de handebol.

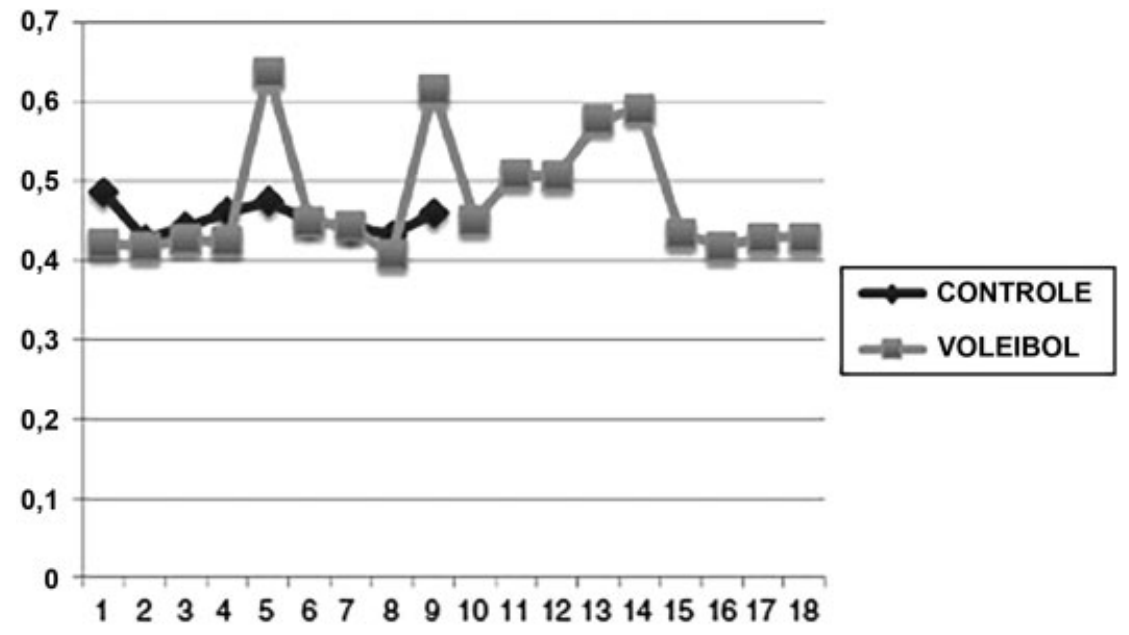

Fig. 3 Comparação dos resultados dos níveis de CTX-II entre o grupo de controle e as jogadoras de voleibol. 
514 Diferença na degeneração articular de acordo com o tipo de esporte Jorge et al.

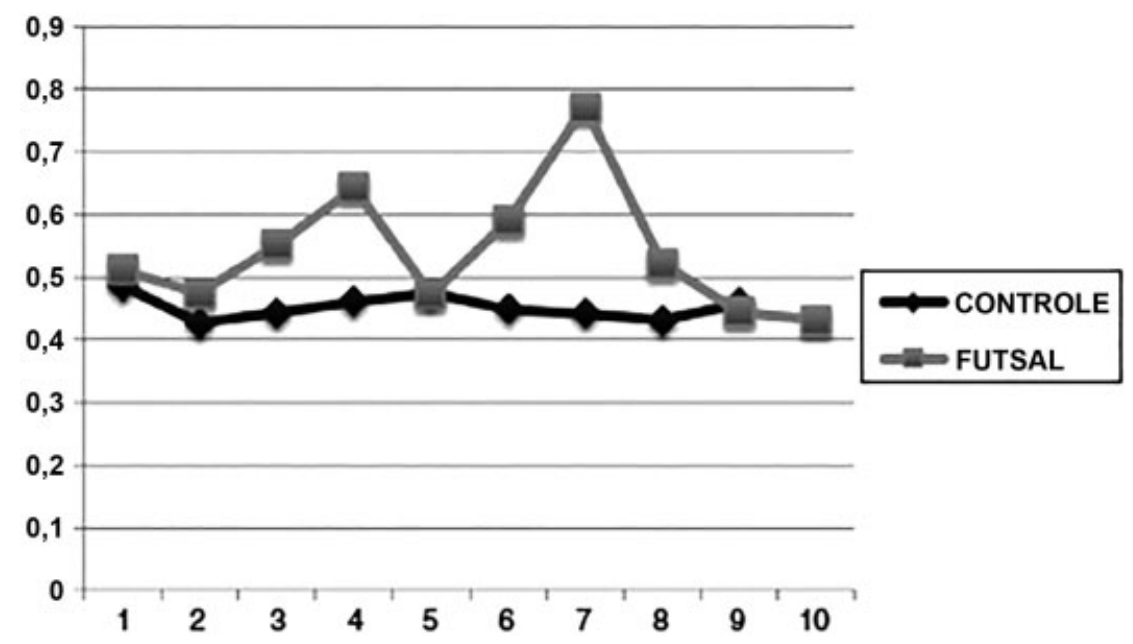

Fig. 4 Comparação dos resultados dos níveis de CTX-II entre o grupo de controle e as jogadoras de futsal.

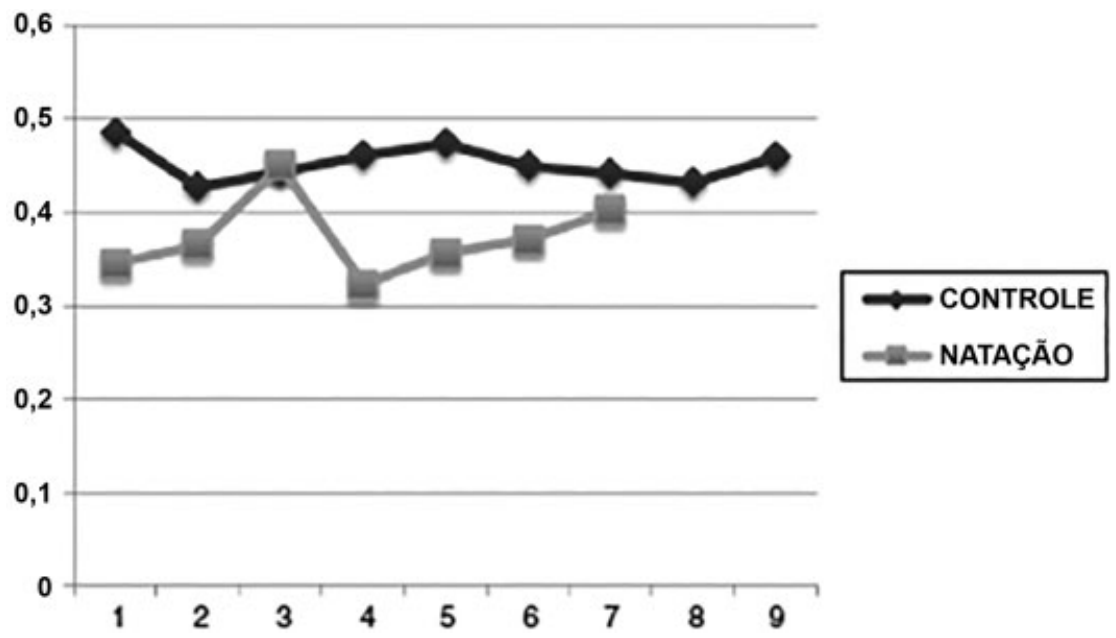

Fig. 5 Comparação dos resultados dos níveis de CTX-II entre o grupo de controle e as nadadoras.

suma importância. No caso dos atletas, o diagnóstico precoce é ainda mais importante, porque sua ocupação depende diretamente da saúde de suas articulações.

Como a primeira clivagem do colágeno tipo II articular catalisada pelas colagenases libera o epítopo CTX-II e este pode ser medido no sangue, na urina e no líquido sinovial, temos uma ferramenta poderosa para o diagnóstico precoce do desgaste articular.

O colágeno tipo II está presente em todas as articulações sinoviais; no entanto, a concentração de CTX-II é maior em pacientes com artrose do joelho e do quadril em comparação com a população geral. ${ }^{27}$ O CTX-II é um biomarcador para a destruição da cartilagem, e um nível aumentado é um preditivo positivo para a redução do espaço articular. $^{27,28}$

Quando medimos o CTX-II em atletas de diferentes modalidades esportivas, procuramos detectar se o risco de sobrecarga e destruição articular precoce seria maior em qualquer esporte em particular. Embora o tamanho da amostra para cada modalidade possa parecer pequeno, trata-se de equipes fechadas, submetidas à mesma carga de treinamento e, em tese, à mesma sobrecarga articular.
Em nossa população de estudo, as jogadoras de voleibol, handebol e futebol não apresentaram maior degradação do colágeno tipo II, apesar de terem participado intensamente de atividades de alto impacto. Talvez esse resultado seja explicado pelo fato de a população do estudo ser muito jovem, e de, nessas três modalidades, o terreno ou os calçados conseguirem absorver o choque.

A degradação do colágeno tipo II foi maior entre as jogadoras de futsal, e foi significativamente diferente do grupo de controle (fig. 4) usando um IC95\%. Essa discrepância era provável porque esse esporte é praticado em um piso rígido, usa sapatos que não absorvem o choque, e tem frequentes mudanças na sobrecarga de direção nas articulações, especialmente nos joelhos (movimentos de pivô repetidos).

Outro resultado notável é que a equipe de natação apresentou valores de CTX-II significativamente menores em comparação ao grupo de controle (-Fig. 5). Em outras palavras, a natação foi um fator de proteção para o colágeno articular na população investigada. Este resultado é provavelmente devido a uma combinação de fatores conhecidos por proteger as articulações, incluindo exercícios aeróbicos, atividade de baixo impacto e fortalecimento dos músculos periarticulares. ${ }^{17}$ 


\section{Conclusão}

Portanto, concluímos que, na população investigada, o treinamento de futsal profissional é um fator de risco para a degradação do colágeno tipo II, e, portanto, de degradação da cartilagem articular. Em contraste, a natação é um fator de proteção para as articulações nessa mesma população, resultando em menor degradação articular do colágeno.

Tais dados sugerem que alguns esportes podem de fato levar à degradação articular precoce. Os médicos traumatologistas do esporte devem considerar este fato, e os protocolos clínicos voltados para a proteção articular devem ser estudados e aplicados.

No entanto, os resultados deste estudo apontam que alguns esportes podem fornecer proteção articular, sugerindo uma ferramenta importante para prevenir a erosão articular, e o treinamento misto pode ser altamente benéfico para equipes profissionais.

Mais estudos sobre este assunto devem ser realizados com amostras maiores, e devem ter como objetivo o controle da destruição articular utilizando meios farmacológicos e não farmacológicos.

Além disso, o uso de biomarcadores de destruição articular é uma ferramenta importante para a detecção precoce da sobrecarga articular. Aqueles que trabalham em traumas esportivos precisam diagnosticar as lesões articulares precocemente para melhorar a vida dos atletas.

Conflitos de Interesse

Os autores declaram não haver conflitos de interesse.

\section{Referências}

1 Martel-Pelletier J, Boileau C, Pelletier JP, Roughley PJ. Cartilage in normal and osteoarthritis conditions. Best Pract Res Clin Rheumatol 2008;22(02):351-384

2 Yoshimura M, Sakamoto K, Tsuruta A, et al. Evaluation of the effect of glucosamine administration on biomarkers for cartilage and bone metabolism in soccer players. Int J Mol Med 2009;24(04):487-494

3 Arokoski JP, Hyttinen MM, Lapveteläinen T, et al. Decreased birefringence of the superficial zone collagen network in the canine knee (stifle) articular cartilage after long distance running training, detected by quantitative polarised light microscopy. Ann Rheum Dis 1996;55(04):253-264

4 Arokoski J, Jurvelin J, Kiviranta I, Tammi M, Helminen HJ. Softening of the lateral condyle articular cartilage in the canine knee joint after long distance (up to $40 \mathrm{~km} /$ day) running training lasting one year. Int J Sports Med 1994;15(05):254-260

5 Arokoski JP, Jurvelin JS, Väätäinen U, Helminen HJ. Normal and pathological adaptations of articular cartilage to joint loading. Scand J Med Sci Sports 2000;10(04):186-198

6 Arokoski J, Kiviranta I, Jurvelin J, Tammi M, Helminen HJ. Longdistance running causes site-dependent decrease of cartilage glycosaminoglycan content in the knee joints of beagle dogs. Arthritis Rheum 1993;36(10):1451-1459

7 Buckwalter JA. Evidence for overuse/overloading of joints in the genesis and progression of osteoarthritis. Curr Orthop 1996;10 (04):220-224

8 Buckwalter JA, Martin JA. Sports and osteoarthritis. Curr Opin Rheumatol 2004;16(05):634-639
9 Coimbra IB, Pastor EH, Greve JM, Puccinelli ML, Fuller R, Cavalcanti FS. Consenso brasileiro para o tratamento da osteoartrite (artrose). [Brazilian consensus for treatment of osteoarthritis (arthrosis)]Rev Bras Reumatol 2002;42(06):371-374

10 Dye SF. The pathophysiology of patellofemoral pain: a tissue homeostasis perspective. Clin Orthop Relat Res 2005;426(436):100-110

11 Hilliquin P. Le Sport chez l'arthrosique. Rev Rhum 2007;74:587-591

12 Luepongsak N, Amin S, Krebs DE, McGibbon CA, Felson D. The contribution of type of daily activity to loading across the hip and knee joints in the elderly. Osteoarthritis Cartilage 2002;10(05): 353-359

13 Oettmeier R, Arakoski J, Roth AJ. Subchondral bone and articular cartilage responses to long distance running training ( $40 \mathrm{~km}$ per day) in the beagle knee joint. Eur J Exp Musculoskel Res. 1992;1:145-154

14 Rezende UM, Gobbi RG. Tratamento medicamentoso da osteoartrose do joelho. Rev Bras Ortop 2009;44(01):14-19

15 Rolland Y, Glisezinski I, Crampes F, Pillard F, Rivière D. Sports et arthrose fémorotibiale. Sci Sports 2000;15:125-132

16 Venn MF. Chemical composition of human femoral and head cartilage: influence of topographical position and fibrillation. Ann Rheum Dis 1979;38(01):57-62

17 Zhang W, Moskowitz RW, Nuki G, et al. OARSI recommendations for the management of hip and knee osteoarthritis, Part II: OARSI evidence-based, expert consensus guidelines. Osteoarthritis Cartilage 2008;16(02):137-162

18 Rodeo SA, Hidaka C, Maher SA. What's new in orthopaedic research. J Bone Joint Surg Am 2005;87(10):2356-2365

19 Bruyere O, Collette J, Kothari M, et al. Osteoarthritis, magnetic resonance imaging, and biochemical markers: a one year prospective study. Ann Rheum Dis 2006;65(08):1050-1054

20 Conrozier T, Balblanc JC, Richette P, et al; Osteoarthritis Group of the French Society of Rheumatology. Early effect of hyaluronic acid intra-articular injections on serum and urine biomarkers in patients with knee osteoarthritis: An open-label observational prospective study. J Orthop Res 2012;30(05):679-685

21 Dam EB, Loog M, Christiansen C, et al. Identification of progressors in osteoarthritis by combining biochemical and MRI-based markers. Arthritis Res Ther 2009;11(04):R115

22 Duclos ME, Roualdes O, Cararo R, Rousseau JC, Roger T, Hartmann DJ. Significance of the serum CTX-II level in an osteoarthritis animal model: a 5-month longitudinal study. Osteoarthritis Cartilage 2010;18(11):1467-1476

23 Felson DT, Lohmander LS. Whither osteoarthritis biomarkers? Osteoarthritis Cartilage 2009;17(04):419-422

24 Huebner JL, Kraus VB. Assessment of the utility of biomarkers of osteoarthritis in the guinea pig. Osteoarthritis Cartilage 2006;14 (09):923-930

25 Ishijima M, Watari T, Naito K, et al. Relationships between biomarkers of cartilage, bone, synovial metabolism and knee pain provide insights into the origins of pain in early knee osteoarthritis. Arthritis Res Ther 2011;13(01):R22

26 Karsdal MA, Byrjalsen I, Bay-Jensen AC, Henriksen K, Riis BJ, Christiansen C. Biochemical markers identify influences on bone and cartilage degradation in osteoarthritis-the effect of sex, Kellgren-Lawrence (KL) score, body mass index (BMI), oral salmon calcitonin (sCT) treatment and diurnal variation. BMC Musculoskelet Disord 2010;11:125

27 Mazières B, Garnero P, Guéguen A, et al. Molecular markers of cartilage breakdown and synovitis at baseline as predictors of structural progression of hip osteoarthritis. The ECHODIAH Cohort. Ann Rheum Dis 2006;65(03):354-359

28 Sowers MF, Karvonen-Gutierrez CA, Yosef M, et al. Longitudinal changes of serum COMP and urinary CTX-II predict X-ray defined knee osteoarthritis severity and stiffness in women. Osteoarthritis Cartilage 2009;17(12):1609-1614 